\title{
Comportamento de Crianças e de Dentistas em Atendimentos Odontológicos Profiláticos e de Emergência
}

\author{
Daniele Pedrosa Fioravante \\ Maria Luiza Marinho-Casanova
}

Universidade Estadual de Londrina

\begin{abstract}
RESUMO
Odontopediatras usam diferentes estratégias para manejo do comportamento infantil durante a consulta, mas aproximadamente um terço das crianças apresentam oposição ao atendimento odontológico. A presente pesquisa visou caracterizar comportamentos de odontopediatras e de crianças durante atendimentos odontológicos. Participaram dois graduados em odontologia e vinte crianças, sendo dez atendidas em profilaxia e dez em emergência. Os dados foram coletados em clínica-escola odontológica através de gravação em vídeo dos atendimentos e categorização dos comportamentos de dentistas e crianças. $40 \%$ das crianças apresentaram alta frequência de respostas opositoras, sendo a oposição infantil mais frequente nos atendimentos de emergência do que nos de profilaxia. Os odontopediatras emitiram, no geral, mais respostas adequadas do que inadequadas, inclusive com $75 \%$ das crianças opositoras; as respostas inadequadas foram emitidas nos atendimentos de emergência. Conclui-se que o tipo de atendimento tem influência sobre o comportamento de crianças e dentistas.
\end{abstract}

Palavras-chave: odontopediatria; comportamento; crianças; colaboração.

\section{ABSTRACT \\ Children's and Dentists' Behavior During Prophylaxis and Emergency Dental Attendances}

Pediatric dentists use different strategies to deal with children's behavior during dental visits. This research intended to characterize the behaviors of the pediatric dentist and of the children during the dental visit. Two pediatric dentists and twenty children were requested to take part in the research. Ten of these children were attended during preventative visits and ten during emergencies. The data were collected in a school-clinic of pediatric dentistry through videos and the categorization of the dentist's and children's behavior. Of the children, $40 \%$ presented a high frequency of opponent responses, which were most frequent on the emergency attendances, comparing with the preventative visits. The pediatric dentist issued, in general, more adequate responses than inadequate responses, even with $75 \%$ of the opponent children; the inadequate responses were presented on the emergency attendances. It was concluded that the kind of attendance exerts influence on the dentist's and children's behavior.

Keywords: pediatric dentistry; behavior; children; cooperation.

A falta de cuidados buco-dentários, principalmente infantis, pode contribuir para a instalação de algumas patologias graves, tais como a cárie dentária, a doença periodontal ou as ulcerações orais recorrentes (Azul \& Trancoso, 2006). Essa falta de cuidados talvez colabore para que o tratamento destes problemas se torne muito mais aversivo para os pacientes, além de onerar bastante os custos dos órgãos públicos com as ações de promoção da saúde bucal da população. Reconhecendo tais prejuízos, as políticas públicas de saúde têm disponibilizado o acesso a atendimentos odontológicos gratuitos. Tal acesso é viabilizado, sobretudo, no que se refere à prevenção do aparecimento de problemas bucais. Alguns exemplos de ações preventivas, são serviços que incluem orientação a pais sobre a importância de se iniciar desde bebê a atenção odontológica, controle da alimentação noturna, intervenção para reduzir hábitos como uso da chupeta e da mamadeira e orientações sobre medidas de higiene bucal (Eaton, McTigue, Fields \& Beck, 2005). Além destes, 
outro serviço preventivo ofertado é o atendimento odontológico denominado de profilaxia, em oposição aos procedimentos odontológicos curativos, que são designados de emergenciais.

Apesar dessa democratização do acesso aos cuidados odontológicos, muitas pessoas ainda evitam se submeter ao tratamento. Segundo Thom, Sartory e Johren (2000), cerca de $20 \%$ da população mundial se esquiva das consultas odontológicas periódicas. No que se refere à população infantil, que muitas vezes é levada à clínica por seus responsáveis, verifica-se uma topografia diferenciada de respostas de fuga e esquiva. Assim, as respostas infantis de fuga e esquiva abrangem outros comportamentos diferentes de não ir à consulta, tais como recusar-se a abrir a boca, levantarse da cadeira odontológica, chorar, gritar, executar movimentos com o corpo ou com a cabeça e, ainda, chutar ou morder o odontopediatra durante os atendimentos. Estes comportamentos recebem, na literatura, a denominação de opositores, constituindo uma das maiores dificuldades do odontopediatra no manejo do comportamento das crianças em situação de atendimento odontológico (Moraes, Sanches, Possobon \& Costa, 2004).

Allen, Stanley e Mc Pherson (1990) apontam que cerca de $25 \%$ das crianças apresentam respostas de oposição, o que constitui frequência relativamente alta, quando se consideram os problemas decorrentes da emissão deste padrão de comportamento no contexto odontológico: aumento no número e na duração das consultas odontológicas, bem como a não execução do tratamento dentário de maneira adequada, o que pode contribuir para piorar a condição bucal. Além disso, para os odontopediatras, o manejo de crianças opositoras pode ser mais difícil.

Visando controlar a emissão de comportamentos opositores das crianças durante a consulta odontológica, alguns profissionais adotam estratégias positivas para o manejo dos comportamentos infantis, como por exemplo: a) modelação de comportamento com uso de filme, que consiste na exposição da criança a um filme, no qual o paciente odontológico apresenta os comportamentos que se espera que a criança adquira por imitação (Melamed, Hawes, Heiby \& Glick, 1975); b) reforçamento de comportamentos adequados, no qual as respostas compatíveis com a execução do procedimento odontológico recebem elogios ou reforços arbitrários por parte do profissional, enquanto as queixas verbais, o choro ou a resistência física, por exemplo, são postos em extinção (Allen, Stark, Rigney, Nash \& Stokes, 1988); c) distração, caracterizada pela apresentação de estímulos incompatíveis com a situação odontológica, de modo que estes adquiram controle sobre o comportamento da criança, diminuindo a frequência de comportamentos não colaborativos (Stark e cols., 1989); e d) fuga contingente $^{1}$ que consiste em interromper o tratamento odontológico por alguns segundos para que a criança descanse, após manifestações de colaboração (Allen, Loiben, Allen \& Stanley, 1992). Alguns autores (Weinstein, 1986; Weinstein, Getz, Ratener \& Domoto, 1982) também observaram que o fornecimento prévio de informação, o direcionamento da conduta e a demonstração de compreensão das reações emocionais das crianças facilitam a aquisição e a manutenção de comportamentos cooperativos em relação ao tratamento, ao passo que diminuem as reações indicativas de medo, como chorar, gritar ou protestar.

Entretanto, há também odontopediatras que defendem o uso de estratégias aversivas como forma rápida e eficaz de se garantir a colaboração infantil. Dentre as estratégias aversivas empregadas pelos dentistas na tentativa de modificar o comportamento das crianças estariam, por exemplo, o controle pela voz (Tambellini, 2005), a restrição física (Guedes-Pinto, Corrêa \& Giglio, 1991), e a estratégia de mão sobre a boca (HOM- hand over mounth) (Kemp, 2005; Moraes, 1999).

Essa postura reflete uma preocupação centrada nos dentes do paciente, em conjunto com a crença de que outra abordagem poderia desviar o profissional de sua tarefa principal (Allen e cols., 1990; Moraes, 1999). Apesar de ser a técnica mais comum de controle do comportamento, é importante ressaltar que a punição, geralmente, acarreta apenas a supressão imediata do comportamento e evoca reflexos característicos de medo, de ansiedade e de outras emoções (Skinner, 1953/1998).

Tais considerações, juntamente com discussões na sociedade acerca dos aspectos éticos e legais implicados na utilização de estratégias aversivas, possivelmente estejam envolvidas na diminuição paulatina no seu emprego por parte dos odontopediatras (Brandenburg, Gavazzoni \& Marinho, 2007). Na década de oitenta, a American Academy of Pediatric Dentistry (1996) constatou, por meio de questionários, que $90 \%$ dos odontopediatras utilizavam algum tipo de estratégia aversiva de controle do comportamento infantil. Em 1990, uma pesquisa similar realizada por Allen e cols. (1990) verificou que esta porcentagem decresceu para $80 \%$, e que $70 \%$ desses profissionais estavam cientes dos aspectos éticos, legais e dos riscos para a saúde, associados a estas práticas. Apesar 
deste decréscimo, as estratégias aversivas continuam a ser largamente empregadas.

Os profissionais da Odontologia interessados no manejo do comportamento infantil têm buscado subsídios junto à Psicologia no intuito de definir um conjunto de estratégias para lidar com pacientes que apresentam dificuldades ao enfrentar um tratamento odontológico (Allen e cols., 1990). A área da Psicologia que realiza estudos para a solução de problemas enfrentados na Odontologia, em especial na Odontopediatria, é denominada Odontologia Comportamental. Pode-se considerar a Odontologia Comportamental como um corpo de conhecimentos útil à avaliação e à manipulação do repertório de comportamentos de pacientes, em especial de crianças, expostos à tratamentos odontológicos. $\mathrm{O}$ objetivo de suas pesquisas e de suas intervenções é contribuir para a promoção e a manutenção do estado geral de saúde do paciente odontológico e facilitar que este enfrente de forma eficiente as situações de tratamento dos transtornos bucais (Moraes \& Pessoti, 1985; Moraes, Possobon \& Ortiz, 2000).

A produção de conhecimentos relevantes para manejo do comportamento infantil durante a consulta odontológica pode ser realizada através da identificação de variáveis contextuais que tenham função para a aquisição e a manutenção dos comportamentos dos profissionais e das crianças naquele contexto (MarinhoCasanova, Brandenburg, Kuniyoshi \& Serafim, 2008). Diante do exposto, a presente pesquisa visou descrever comportamentos de odontopediatras e de crianças durante atendimentos odontológicos.

\section{MÉTODO}

Participaram dois profissionais graduados em odontologia, há um ano, no início da pesquisa: OP1, sexo masculino, 25 anos de idade e OP2, sexo feminino, 26 anos de idade. Os profissionais cursavam Especialização em Odontopediatria e formavam dupla durante o estágio na clínica-escola.

Houve, ainda, a participação de 20 crianças (C1 a C20), pacientes de um Núcleo de Odontologia para Bebês que presta atendimento gratuito à população. As crianças eram de ambos os sexos, com idades entre 18 meses e seis anos; nenhuma delas foi indicada como apresentando problemas de comportamento, de acordo com os resultados obtidos no inventário para avaliação do comportamento infantil Walker Problem Behavior Identification Checklist $(1976)^{2}$, respondido pela mãe.

Cada profissional atendeu a 10 crianças: cinco casos para realização de procedimentos profiláticos e outros cinco em situação de emergência. Os cuidados profiláticos englobavam procedimentos como a escovação, o uso do fio dental, a aplicação de flúor e a observação da condição dentária geral. Segundo Rape e Bush (1994), os procedimentos odontológicos de emergência são, geralmente, mais dolorosos, pois implicam penetração e/ou introdução de agulhas, bisturis e outros instrumentos odontológicos no tecido bucal ou restrição dos movimentos corporais normais. A Tabela 1 apresenta os procedimentos de emergência a que cada criança foi submetida.

TABELA 1

Descrição dos Procedimentos de Emergência Realizados e as Crianças a Eles Submetidas nas Consultas Observadas

\begin{tabular}{llc}
\hline Procedimento Odontológico & Descrição & Crianças submetidas \\
\hline Pedoforme & $\begin{array}{l}\text { Colocação de coroa dentária de metal ou acrílico em dentes parcialmente ou } \\
\text { totalmente destruídos por cáries. }\end{array}$ & C6, C20 \\
\hline Aplicação de selantes & $\begin{array}{l}\text { Selamento dos sulcos e estruturas que possam reter restos alimentares na } \\
\text { superfície do dente. }\end{array}$ & C7 \\
\hline Extração dentária & Remoção cirúrgica de um dente. & C8, C19 \\
\hline Colocação de borracha para & Procedimento que ajuda na expulsão do dente, amolecendo-0. & C9 \\
esfoliação dental & & C10 \\
\hline Colocação de ionômero de vidro & Procedimento para restauração estética de dentes com defeito de estrutura. & C16 \\
\hline Massa para aparelho & Procedimento no qual se retira o molde do dente para a posterior confeç̧ão & C17 \\
\hline Endodontia de dentes decíduos & Te aparelho ortodôntico. & C18 \\
\hline Choque & Aplicação de flúor (safluoraide) em todos os dentes & C18, C19 \\
\hline Adequação & Consiste em limpar o dente cariado, colocando um curativo para aguardar 0 & C19 \\
Aplicação de carióstico & tratamento. & Medicamento que visa estacionar o processo de cárie. \\
\hline
\end{tabular}

Nota. Fonte da descrição: INPAO - Instituto de Previdência e Assistência Odontológica (2000). 
Todos os atendimentos foram gravados em vídeo para posterior análise. Duas observadoras que não participaram das filmagens efetuaram a categorização das respostas apresentadas pelos odontopediatras e pelas crianças durante a realização dos atendimentos odontológicos. As categorias comportamentais adotadas foram estabelecidas previamente e estão descritas na Tabela 2.

\section{TABELA 2}

Categorias de Resposta de Odontopediatras e de Crianças em Consulta Odontológica (Adaptado de Fioravante e cols., 2007)

\section{CATEGORIAS PARA AVALIAÇÃO DAS RESPOSTAS DOS ODONTOPEDIATRAS}

\begin{tabular}{lll}
\hline Categorias de resposta & Descrição & Exemplos \\
\hline \multirow{4}{*}{ ADEQUADOS } & Aqueles que podem tornar o contexto odontológico, & - Aperta o nariz para a cadeira mágica subir. \\
& em geral, mais reforçador para a criança, tais como: & - Agora eu vou pegar o Bob Esponja! \\
& elogiar, fantasiar, descrever o funcionamento do & - Eu sei que você deve estar com medo, mas não vai \\
& equipamento, oferecer conforto ou contato, distrair & doer nada, tá? \\
& ou direcionar. & - Que bom que você ficou quietinha! \\
\hline INADEQUADOS & Aqueles que podem tornar o contexto odontológico, & - Tá doido! \\
& em geral, mais aversivo para a criança, tais como: & - Que coisa feia, chorando deste tamanho! \\
& criticar, imobilizar ou restringir fisicamente, restringir & - Não morde o dedo da tia. \\
& verbalmente, deixar a criança sozinha ou falar com & - Olha, você ta vendo este dente aqui? (dirigido para a \\
& outras pessoas como se ela não estivesse presente & mãe). \\
& ou fazer comentários não condizentes com a situa- & - Que lindo! (quando a criança está imobilizada e cho- \\
ção. & rando muito). \\
\hline \hline
\end{tabular}

\section{CATEGORIAS PARA AVALIAÇÃO DAS RESPOSTAS DAS CRIANÇAS}

\begin{tabular}{lll}
\hline Categorias de resposta & Descrição & Exemplos \\
\hline \multirow{4}{*}{ COOPERATIVOS } & Aqueles que podem tornar o contexto odontológico, & - Também tem uma escova lá na minha casa! \\
& em geral, mais reforçador para o odontopediatra, & - Para quê serve isso? \\
& tais como: permitir a realização dos procedimentos & - Deixa que eu seguro a girafa! \\
& da consulta, solicitar informação, responder, olhar, & - Você vai lembrar a tia de te dar um dese- \\
& buscar suporte emocional, auxiliar. & nho?(profissional) \\
& & Sim ! (criança) \\
\hline \multirow{2}{*}{ OPOSITORES } & Aqueles que podem tornar o contexto odontológico & - Anh, anh, anh! \\
& em geral mais aversivo para o odontopediatra, tais & - Pára! \\
& como chorar, gritar, agredir, fugir, movimentar-se, & - Tá doendo! \\
\hline
\end{tabular}

Nota. Para o registro do número de respostas que ocorriam a cada minuto, foi pré-estabelecido que: 1) em categorias que se referem a respostas verbais, nova ocorrência foi contada a cada pausa de no mínimo dois segundos; 2) respostas motoras: nova ocorrência a cada nova movimentação; 3) imobilizar ou restringir fisicamente e choro: foram cronometrados e cada cinco segundos de choro/choramingo ou de restrição/ imobilização física era considerado como a emissão de uma resposta.

A duração das consultas odontológicas não era fixa (variavam de três minutos a cerca de 40 minutos). No caso de consultas mais longas, restringiu-se a categorização ao tempo de 30 minutos. Categorizações além deste período, realizadas em estudo piloto, demonstraram não alterar o padrão observado nos primeiros 30 minutos.

Registrou-se a frequência de ocorrência de cada uma das categorias de resposta dos odontopediatras e das crianças em cada minuto da consulta. O registro dos comportamentos da criança e do odontopediatra foram feitos separadamente. $\mathrm{O}$ dado numérico anali- sado foi a frequência média por minuto, conforme a Tabela 3.

\section{RESULTADOS}

A frequência média de respostas adequadas e inadequadas por minuto, emitidas por OP1 e OP2 durante o atendimento das 20 crianças é apresentada na Tabela 3. Nessa mesma Tabela é apresentada a frequência média por minuto de respostas cooperativas e de respostas opositoras emitidas pelas crianças durante as consultas. 
TABELA 3

Frequência Média das Respostas dos Odontopediatras e das Crianças Durante os Atendimentos de Profilaxia e de Emergência

\begin{tabular}{|c|c|c|c|c|c|c|c|c|c|}
\hline \multicolumn{10}{|c|}{ Observações dos atendimentos conduzidos pelo odontopediatra OP1 } \\
\hline \multirow[t]{2}{*}{ Profilaxia } & \multicolumn{4}{|c|}{ Frequência média das Respostas/minuto } & Emergência & \multicolumn{4}{|c|}{ Frequência média das Respostas/minuto } \\
\hline & \multicolumn{2}{|c|}{ Odontopediatra } & \multicolumn{2}{|c|}{ Criança } & & \multicolumn{2}{|c|}{ Odontopediatra } & \multicolumn{2}{|c|}{ Criança } \\
\hline Díades & Adeq & Inad & Coop & Opos & Díades & Adeq & Inad & Coop & Opos \\
\hline OP1xC1 & 1,75 & 0,1 & 3,85 & 0,75 & OP1xC6 & 1 & 1 & 1,5 & 0 \\
\hline $\mathrm{OP} 1 \times \mathrm{x} 2$ & 3,5 & 0,3 & 2,85 & 0,95 & OP1xC7 & 1,32 & 1,7 & 1,11 & 0,4 \\
\hline $\mathrm{OP} 1 \times \mathrm{CC} 3$ & 2,4 & 0,6 & 3,3 & 0,2 & OP1xC8 & 2,17 & 4,88 & 3,02 & 9,42 \\
\hline $\mathrm{OP} 1 \times \mathrm{C} 4$ & 5,54 & 0,88 & 2,15 & 5,91 & OP1xC9 & 4,33 & 0 & 3,49 & 0 \\
\hline $\mathrm{OP} 1 \times \mathrm{C} 5$ & 4,4 & 0,2 & 3,4 & 0 & $\mathrm{OP} 1 \times \mathrm{C} 10$ & 0,44 & 0,7 & 1,42 & 0,37 \\
\hline Média & 3,25 & 0,42 & 3,11 & 1,56 & Média & 1,85 & 1,66 & 2,11 & 2,04 \\
\hline \multicolumn{10}{|c|}{ Observações dos atendimentos conduzidos pela odontopediatra OP2 } \\
\hline Profilaxia & \multicolumn{4}{|c|}{ Frequência média das Respostas/minuto } & Emergência & \multicolumn{4}{|c|}{ Frequência média das Respostas/minuto } \\
\hline & \multicolumn{2}{|c|}{ Odontopediatra } & \multicolumn{2}{|c|}{ Criança } & & \multicolumn{2}{|c|}{ Odontopediatra } & \multicolumn{2}{|c|}{ Criança } \\
\hline Díades & Adeq & Inad & Coop & Opos & Díades & Adeq & Inad & Coop & Opos \\
\hline OP2xC11 & 4,35 & 0,6 & 1,4 & 0 & OP2xC16 & 1 & 1 & 2 & 0 \\
\hline $\mathrm{OP} 2 \mathrm{xC} 12$ & 2,43 & 0,94 & 4,2 & 0,6 & $\mathrm{OP} 2 \times \mathrm{C} 17$ & 1,73 & 5,06 & 0,49 & 21,7 \\
\hline $\mathrm{OP} 2 \mathrm{xC} 13$ & 8,08 & 0,22 & 3,92 & 3,65 & $\mathrm{OP} 2 \times \mathrm{C} 18$ & 2,76 & 2,5 & 2,13 & 2,69 \\
\hline OP2xC14 & 6,13 & 0,14 & 2,7 & 0,14 & OP2xC19 & 4,71 & 0,24 & 0,54 & 3,57 \\
\hline OP2xC15 & 8,21 & 0,11 & 4,09 & 2,6 & $\mathrm{OP} 2 \times \mathrm{C} 20$ & 2,68 & 0,89 & 1,3 & 4,83 \\
\hline Média & 5,84 & 0,40 & 3,26 & 1,40 & Média & 2,58 & 1,94 & 1,29 & 6,55 \\
\hline $\begin{array}{l}\text { Média geral } \\
\text { profilaxia }\end{array}$ & 4,54 & 0,41 & 3,18 & 1,48 & $\begin{array}{l}\text { Média geral } \\
\text { emergência }\end{array}$ & 2,21 & 1,80 & 1,70 & 4,29 \\
\hline
\end{tabular}

Nota. OP: Odontopediatra/ C: Criança/ Adeq: Adequadas/ Inad: Inadequadas/ Coop: Cooperativas/ Opos: Opositoras.

Os dados apresentados na Tabela 3 indicam que em 14 dos 20 atendimentos analisados os odontopediatras emitiram mais respostas adequadas do que respostas inadequadas. Quando se comparam os dados observados nos atendimentos da profilaxia e da emergência, observa-se que houve diferenças no que se refere à frequência de emissão de respostas adequadas pelos profissionais. Em todos os 10 atendimentos de profilaxia os profissionais apresentaram mais respostas adequadas do que inadequadas; em contrapartida, nos atendimentos de emergência os profissionais apresentaram mais respostas adequadas do que inadequadas em apenas quatro dos 10 atendimentos.

Diferenças entre as respostas dos odontopediatras nos dois tipos de atendimento também podem ser observadas através da frequencia média total das respostas. Houve mais respostas adequadas dos profissionais nos atendimentos de profilaxia do que nos de emergência. OP1 e OP2 apresentaram, respectivamente, as seguintes médias de respostas adequadas por minuto: 3,25 e 4,54 nos atendimentos da profilaxia, contra 1,85 e 2,21 nos atendimentos de emergência. Em direção inversa, houve mais respostas inadequadas dos profissionais nos atendimentos de emer- gência do que nos de profilaxia. OP1 e OP2 apresentaram, respectivamente, as seguintes médias de respostas inadequadas por minuto: 1,66 e 1,80 na emergência, contra 0,42 e 0,41 na profilaxia.

A análise dos dados de cada atendimento corrobora essa afirmação. Observa-se que as frequências mais elevadas de respostas inadequadas dos profissionais $\left(4,88 \mathrm{r} / \mathrm{min}^{3} ; 5,06 \mathrm{r} / \mathrm{min}\right.$ e $\left.2,5 \mathrm{r} / \mathrm{min}\right)$ ocorreram nos atendimentos a três crianças da emergência (respectivamente $\mathrm{C} 8, \mathrm{C} 17$ e $\mathrm{C} 18$ ). Os procedimentos odontológicos realizados foram: remoção cirúrgica de um dente, tratamento de canal e limpeza de dente cariado (ver Tabela 1). Isso sugere maior dificuldade dos profissionais em realizar esses procedimentos e se manterem comportando de forma reforçadora. Entretanto, é importante destacar que o paciente C19 também foi submetido a esses mesmos procedimentos (ver Tabela 1) e o profissional praticamente não apresentou respostas inadequadas $(0,24 \mathrm{r} / \mathrm{min})$ durante $\mathrm{o}$ atendimento.

Em relação às respostas das crianças, os dados indicaram que a maioria delas (14 das 20 crianças observadas) apresentou mais comportamentos de colaboração do que de oposição à realização do procedimento odontológico. Das seis crianças que apresenta- 
ram mais comportamentos de oposição do que de colaboração, cinco foram atendidas em emergência.

A análise dos dados sugere que não houve relação entre resposta de oposição da criança e repostas inadequadas dos odontopediatras durante os atendimentos. Das 20 crianças atendidas, oito apresentaram mais de uma resposta opositora por minuto $(\mathrm{C} 4, \mathrm{C} 8, \mathrm{C} 13$, C15, C17, C18, C19 e C20). Durante o atendimento de cinco destas oito crianças com respostas opositoras, os odontopediatras apresentaram mais respostas adequadas do que respostas inadequadas $(\mathrm{C} 4, \mathrm{C} 13, \mathrm{C} 15$, C19 e C20). Além disto, a frequência das repostas inadequadas dos odontopediatras com estas cinco crianças foi baixa, entre 0,11 e 0,88 respostas por minuto.

Esses dados comentados anteriormente sugerem que tanto as respostas dos odontopediatras como das crianças foram afetadas por variáveis relacionadas ao tipo de atendimento. Podem-se observar, também, algumas diferenças no padrão de atendimento de ambos profissionais. Como se pode observar na Tabela 3, o profissional do sexo feminino (OP2) apresentou maior frequência total de respostas adequadas por minuto do que o profissional do sexo masculino (OP1), tanto na profilaxia $(4,54 \mathrm{r} / \mathrm{min}$ contra $3,25 \mathrm{r} / \mathrm{min}$, respectivamente) como na emergência $(2,21 \mathrm{r} / \mathrm{min}$ contra $1,85 \mathrm{r} / \mathrm{min}$ ). Essas diferenças não pareceram estar em função do comportamento infantil, já que o profissional com mais respostas adequadas (OP2) foi quem atendeu a maioria das crianças que apresentaram mais respostas opositoras $(\mathrm{C} 13, \mathrm{C} 15, \mathrm{C} 17, \mathrm{C} 18$, C19 e C20).

\section{DISCUSSÃO}

A porcentagem das crianças observadas no presente estudo, que apresentaram maior frequência de respostas opositoras do que colaboradoras, foi de $30 \%$ (seis crianças), um pouco superior aos $25 \%$ apontados na literatura (Allen e cols., 1990). Supõe-se que o tamanho da amostra possa ter contribuído para essa discrepância nos dados, sendo importante analisar as respostas de um número maior de crianças brasileiras, para se comparar com dados da literatura internacional.

Alguns autores (por exemplo, Fioravante, Soares, Silveira \& Zakir, 2007; Rolim, Moraes, César \& Costa, 2004) sugerem que as respostas de oposição de crianças à realização do procedimento odontológico podem dificultar o manejo do comportamento infantil por parte dos odontopediatras; e que na tentativa de concluir a execução do procedimento, os profissionais podem apresentar muitas respostas inadequadas durante a interação com a criança. Os dados do presente estudo, entretanto, sugerem que as respostas dos profissionais foram mais influenciadas pelo tipo de pro- cedimento odontológico do que pelas respostas das próprias crianças atendidas. Isto pode ser observado pelo fato dos odontopediatras emitirem respostas adequadas com cinco das oito crianças que apresentaram muitas respostas de oposição ao procedimento odontológico, como comentado anteriormente. Além disto, os odontopediatras emitiram mais respostas adequadas do que inadequadas com crianças opositoras da profilaxia.

É possível que a complexidade de execução de certos procedimentos odontológicos na emergência tenha dificultado o manejo adequado do comportamento infantil. Isso pode ser justificado pelo fato dos profissionais participantes da pesquisa estarem em treinamento no atendimento de crianças, adicionado do fato de que a Odontopediatria é ainda um ramo de conhecimentos relativamente recente (Moraes, 1999). Talvez, por sua pouca prática, tenha sido mais difícil para os profissionais realizarem procedimentos odontológicos complexos, ao mesmo tempo em que conversavam, elogiavam ou distraiam as crianças, por exemplo. Tentativas de manejo do comportamento opositor infantil pelo odontopediatra através de estratégias adequadas podem não ter sido reforçadas, ou mesmo terem sido punidas durante os atendimentos de emergência, de modo a diminuírem de frequência no que se refere ao repertório de comportamentos desses profissionais. Além disso, as respostas inadequadas (como conter fisicamente crianças opositoras) podem ter aumentado de frequência pelo seu efeito mais imediato (no caso, reduzir movimentação física da criança e permitir a conclusão do procedimento).

Por outro lado, a adequada interação entre os profissionais e os pacientes nos atendimentos da profilaxia sugere a existência de esquema de reforçamento positivo de mão dupla. Segundo alguns autores (Prins, Veerkamp, Horst, Jong \& Tan, 1987; Weinstein 1986; Weinstein e cols., 1982), a alta frequência de respostas adequadas e a baixa frequência de respostas inadequadas de odontopediatras em atendimentos de profilaxia podem facilitar a aquisição e a manutenção dos padrões cooperativos infantis. Destaca-se o fato de que, no presente estudo, as crianças atendidas na profilaxia geralmente foram acompanhadas no Programa Educativo-Preventivo da clínica-escola desde os três meses de idade, possivelmente, obtendo treino anterior em relação a este contexto.

Nessas condições, considera-se que: a) as respostas dos profissionais de elogiar e de acariciar as crianças durante os atendimentos podem reforçar positivamente as respostas cooperativas; b) as respostas de cooperação infantil podem contribuir para reforçar positivamente as respostas adequadas dos profissionais, aumentando sua probabilidade de emissão durante esse tipo de procedimento odontológico; c) as 
respostas opositoras das crianças podem sofrer extinção durante a profilaxia, pois não têm a função de cessar a execução do procedimento (Fioravante e cols., 2007). Isto pôde ser observado nesta pesquisa ao se verificar que os odontopediatras manejavam as respostas opositoras das crianças mediante estratégias adequadas, sem, no entanto, interromper o procedimento que estava sendo realizado.

Em relação ao comportamento de oposição das crianças, pode-se hipotetizar algumas variáveis antecedentes e consequentes relacionadas. Nos casos das crianças atendidas na emergência, é importante destacar que estas não eram pacientes regulares da clínicaescola, tendo procurado o atendimento devido à queda ou outro fator que tenha afetado a sua condição dentária. Antecedentes, como história anterior de aprendizagem, tipo de procedimento odontológico e novidade da situação, além de consequências como cessar a estimulação aversiva presente, podem manter a frequência de respostas de oposição de crianças (Cardoso \& Loureiro, 2005; Costa, 2002).

Observaram-se, ainda, algumas crianças com muitas respostas opositoras também na profilaxia. Pode-se hipotetizar alguns dos antecedentes que poderiam estar relacionados à emissão das respostas opositoras das crianças nesse tipo de atendimento. Conforme comentado, tais respostas opositoras não pareceram se relacionar às respostas inadequadas dos profissionais, já que estes praticamente não as apresentaram durante a profilaxia. Assim, possivelmente, procedimentos profiláticos tenham sido aversivos para uma parte das crianças. Apesar de serem acompanhadas desde os três meses de idade, algumas crianças experienciaram situações aversivas no contexto odontológico, como contenção física, por exemplo. E uma vez experienciadas tais situações, elas podem contribuir para tornar todo contexto odontológico potencialmente aversivo, mediante o emparelhamento de alguns estímulos, de modo a evocar, posteriormente, respostas de fuga e esquiva.

Conclui-se que as interações de ambos os odontopediatras com as crianças foram caracterizadas, principalmente, pela emissão de respostas adequadas por parte dos profissionais. O número de crianças com respostas de oposição ao atendimento odontológico foram pouco superiores às apresentadas na literatura da área. Variáveis relacionadas ao tipo de atendimento (emergência ou profilaxia) parecem exercer influência tanto sobre o comportamento dos pacientes como dos odontopediatras, sendo os maiores problemas observados nos atendimentos de emergência. As crianças apresentaram mais comportamentos de colaboração na profilaxia e mais comportamentos opositores na emergência, o que indica que o tipo de atendimento interfere no comportamento infantil durante a consul- ta. Os odontopediatras emitiram mais respostas adequadas do que inadequadas com quatro das seis crianças que emitiram mais respostas opositoras do que cooperativas, o que sugere que o comportamento infantil de oposição não foi a principal variável de controle para a apresentação de comportamento inadequado por parte dos profissionais.

A frequência maior de respostas adequadas do profissional do sexo feminino observada no presente estudo indica a relevância de se investigar essa questão com um número maior de odontopediatras. Além disso, a partir dos dados apresentados, sugere-se investigar se melhor treino do odontopediatra na execução de procedimentos odontológicos invasivos tem efeito sobre a frequência de respostas inadequadas por parte dos profissionais.

Com base no exposto, considera-se que duas grandes contribuições da Psicologia para a formação dos odontopediatras seriam o fornecimento de informações sobre os princípios que regem o comportamento humano e o ensino de estratégias comportamentais de manejo do comportamento infantil.

\section{REFERÊNCIAS}

Allen, K. D., Loiben, T., Allen S. J., \& Stanley, R. T. (1992). Dentist-implemented contingent escape for management of disruptive child behavior. Journal of Applied Behavior Analysis, 25, 629-636.

Allen, K. D., Stanley, R., \& McPherson, K. (1990). Evaluation of behavior management technology dissemination in pediatric dentistry. Pediatric Dentistry, 12, 79-82.

Allen, K. D., Stark, L. J, Rigney, B. A., Nash, D. A., \& Stokes, T. F. (1988). Reinforced practice of children's cooperative behavior during restorative dental treatment. Journal of Dentistry for Children, 55, 273-277.

American Academy of Pediatric Dentistry (1996). Guidelines for behavior management. Pediatric Dentistry, 18 (Special issue), 86-88.

Azul, A. M., \& Trancoso, P. F. (2006). Patologia mais frequente da mucosa oral. Revista Portuguesa de Clínica Geral, 2, 369377.

Brandenburg, O. J., Gavazzoni, J. A. \& Marinho, M. L. (2007). Estratégias utilizadas por odontopediatras no manejo do comportamento infantil. Em: R. R. Starling (Org.), Sobre Comportamento e Cognição: Temas aplicados (pp. 265-273). Santo André, SP: Esetec.

Cardoso, C. L., \& Loureiro, S. R. (2005). Problemas comportamentais e stress em crianças com ansiedade frente ao tratamento odontológico. Estudos de Psicologia (Campinas), 22(1), 5-12.

Costa, A. L., Jr. (2002). Psicologia aplicada à odontopediatria: Uma introdução. Estudos e Pesquisas em Psicologia, 2(2), 3-12.

Eaton, J. J., McTigue, D. J., Fields Jr., H. W., \& Beck, F. M. (2005). Attitudes of contemporary parents toward behavior management techniques used in pediatric dentistry. Pediatric Dentistry, 27, 107-113. 
Fioravante, D. P., Soares, M. R. Z, Silveira, J. M., \& Zakir, N. S. (2007). Análise funcional da interação profissional-paciente em odontopediatria. Estudos de Psicologia (Campinas), 24, 267-277.

Guedes-Pinto, A. C., Corrêa, M. S. N. P., \& Giglio, E. M. (1991). Conduta clínica e psicologia em odontologia pediátrica $\left(3^{\mathrm{a}}\right.$ ed.) São Paulo: Livraria Santos.

Kemp, F. (2005). Alternatives: A review of non-pharmacological approaches to increasing the cooperation of patients with special needs to inherently unpleasant dental procedures. The Behavior Analist Today, 6(2), 88-108.

Marinho-Casanova, M. L., Brandenburg, O. J., Kuniyoshi, P. T. \& Serafim, S. B. (2008). Odontopediatria e Comportamento: dados empíricos de uma década de investigação Em: W. C. M. P. da Silva (Org.), Sobre Comportamento e Cognição. Reflexões epistemológicas e conceituais, considerações metodológicas e relatos de pesquisa (pp. 141-153). Santo André, SP: Esetec.

Melamed, B. G., Hawes, R. R., Heiby, E., \& Glick, J. (1975). Use of filmed modeling to reduce uncooperative behavior of children during dental treatment. Journal of Dentistry Research, 54, 797-801.

Meyer, S. B. (1997). O conceito de análise funcional. Em M. Delitti (Org.), Sobre comportamento e cognição: Vol. 2 - A prática da análise do comportamento e da terapia cognitivocomportamental (pp. 31-36). São Paulo: Arbytes.

Moraes, A. B. A. (1999). Comportamento e saúde bucal: Circunscrevendo o campo. Em R. R. Kerbauy (Org.), Comportamento e saúde: Explorando alternativas (pp. 61-83). Santo André, SP: Arbytes.

Moraes, A. B. A., \& Pessotti, L. (1985). Psicologia aplicada à odontologia. São Paulo: Sarvier.

Moraes, A. B. A, Possobon, R. F., \& Ortiz, C. E. (2000). Motivação e comportamento preventivo de saúde bucal em programas de assistência odontopediátrica na primeira infância. Pesquisa Odontológica Brasileira, 14(3), 287-293.

Moraes, A. B. A., Sanchez, K. A. S., Possobon, R. F., \& Costa A., L., Jr. (2004). Psicologia e odontopediatria: A contribuição da análise funcional do comportamento. Psicologia: Reflexão $e$ Crítica, 17(1), 75-82.
Prins, P., Veerkamp, J., Horst, G., Jong, A., \& Tan, L. (1987). Behavior of dentists and child patients during treatment. Community Dentistry of Oral Epidemiology, 15, 253-257.

Rape, R. N., \& Bush, J. P. (1994). Psychological preparation for pediatric oncology patients undergoing painful procedures: A methodological critique of the research. Children's Health Care, 23(1), 51-57.

Rolim, G. S., Moraes, A. B. A., César, J., \& Costa Jr., A. L. (2004). Análise do comportamento do odontólogo no contexto de atendimento infantil. Estudos de Psicologia (Natal), 9(3), 533-541.

Skinner, B. F. (1998). Ciência e comportamento humano (J. C. Todorov \& R. Azzi, Trad.). São Paulo: Martins Fontes.

Stark, L. J., Allen, K. D., Hurst, M., Nash, D. A., Rigney, B., \& Stokes, T. F. (1989). Distraction: Its utilization and efficacy with children undergoing dental treatment. Journal of Applied Behavior Analysis, 22, 297-307.

Tambellini, M. M. (2005). Elaboração e padronização de escala avaliativa do comportamento de crianças em sua primeira consulta odontológica. Dissertação de mestrado não-publicada. Universidade de São Paulo, Ribeirão Preto.

Thom, A., Sartory, G., \& Johren, P. (2000). Comparison between one-session psychological treatment and benzodiazepine in dental phobia. Journal of Consulting and Clinical Psychology, 68, 378-387.

Walker, H. M. (1976). Walker Problem Behavior Identification Checklist Manual (WPBIC). Los Angeles: Western Psychological Service.

Weinstein, P. (1986). Integrating behavioral methodologies into dental pharmacological research. Anesthesia Progress, 33, 55-59.

Weinstein. P., Getz, T., Ratener, P., \& Domoto, P. (1982). The effect of dentist's behaviors on fear-related behaviors in children. Journal of American Dentistry Association, 104, 32-38.

Recebido: $15 / 09 / 2008$

Última revisão: 06/01/2009

Aceite final: 03/02/2009

Notas:

1 Embora seja utilizado na literatura da área o termo "fuga contingente", o que melhor descreve tal procedimento é o termo "pausa contingente".

2 O instrumento é composto por 50 afirmações que descrevem comportamentos de crianças. É utilizado para avaliação do comportamento infantil pelos pais ou responsáveis, através da indicação das afirmações que considerem verdadeiras em relação à criança nos dois últimos meses. Pontuações ponderadas iguais ou superiores a 60 pontos indicam que a criança apresenta problema de comportamento em uma ou mais das áreas mensuradas pelo instrumento: impulsividade, isolamento, atenção instável, relações perturbadas com os pares e imaturidade.

3 Indica a frequência de respostas por minuto.

\section{Sobre as autoras:}

Daniele Pedrosa Fioravante: Graduada em Psicologia pela Universidade Estadual de Londrina, Mestre em Análise do Comportamento pela Universidade Estadual de Londrina.

Maria Luiza Marinho-Casanova: Graduada em Psicologia pela Universidade Estadual de Londrina, Doutora em Psicologia Clínica pela Universidade de São Paulo, Pós-doutorado em Psicologia Clínica pela Universidade de Granada, Espanha.

Endereço para correspondência: Maria Luiza Marinho. Universidade Estadual de Londrina. Campus Universitário. Centro de Ciências Biológicas. Departamento de Psicologia Geral e Análise do Comportamento. Caixa Postal 6001. 86051-990 Londrina - PR. Endereço eletrônico: marinho.malu@hotmail.com. 